\title{
General model for a entanglement-enhanced composed quantum game on a two-dimensional lattice
}

\author{
Jarosław Adam Miszczak and Łukasz Paweld* \\ Institute of Theoretical and Applied Informatics, \\ Polish Academy of Sciences, Battycka 5, 44-100 Gliwice, Poland \\ Jan Sładkowski \\ Institute of Physics, University of Silesia, Uniwersytecka 4, 40-007 Katowice, Poland
}

(Dated: 19/02/2014 (v. 0.20))

\begin{abstract}
We introduce a method of analyzing entanglement enhanced quantum games on regular lattices of agents. Our method is valid for setups with periodic and non-periodic boundary conditions. To demonstrate our approach we study two different types games, namely the prisoner's dilemma game and a cooperative Parrondo's game. In both cases we obtain results showing, that entanglement is a crucial resource necessary for the agents to achieve positive capital gain.
\end{abstract}

PACS numbers: 03.67.-a, 02.50.Le, 05.40.Fb

Keywords: quantum information; decision theory; quantum games; mobile agents; entanglement; quantum walks

\section{INTRODUCTION}

Systems involving a large number of simple variables with mutual interactions appear frequently in various fields of research. Often the interactions are of local character. Network models have proven to be successful in analysis of such phenomena [1. Axelrod 2, 3] and Nowak and May [4] prompted scientist to investigate networks with local interactions that model various social end economic structures. Cooperation and coordination are among the key issues in economics and social sciences and can be analysed from this point of view 5. Game theoretical models often provide a qualitative way of understanding various aspects of human decisions and behaviour of complex systems. The combined network and game theoretical methods resulted in a description of population structures with local interactions with sometimes astonishing accuracy. The fields of econophysics and sociophysics were born during the process [6]. Bearing in mind interesting analyses of a wide spectrum of problems performed from the quantum game theoretical point of view 9 12, one should not be astonished that quantum games have invaded the network territory.

Quantum game theory approach extends such analyses in an interesting way [10 13. Cooperation is usually modeled in the context of Prisoner's dilemma 14 . Another interesting phenomenon is known as Parrondo paradox 15, the counterintuitive fact that in some cases combination of apparently loosing games can result in success. Such games have their quantum counterparts and this spurred us on to the analysis described in the present work.

\footnotetext{
* lukasz.pawela@gmail.com

$\dagger$ jan.sladkowski@us.edu.pl
}

The main contribution of this work is to provide a consistent model allowing to study quantum games on twodimensional lattice. We provide a general ingredients needed in order to implement any quantum game in this general scheme. In particular, we address the problem of using multipartite entangled states shared by players. As a particular case, we study the family of games on two-dimensional lattice constructed using the Parrondo scheme.

This paper is organized as follows. In Section II we provide necessary background and notation. In Section III we develop a general model allowing the incorporation of a strategic quantum games into the evolutionary scheme on two-dimensional graphs. In Section IV we use the introduced model to provide an uniform analysis of a family of quantum games, focusing on the games based on the Parrondo scheme. Finally, in Section $[\mathrm{V}]$ we draw the final conclusions.

\section{PRELIMINARIES}

Strategic game theory studies mathematical models of conflict and cooperation between rational decisionmakers [16 and is widely applied in a great number of fields, ranging from biology to social sciences and economics. Recently, the scientific community realized that quantum phenomena might be important in this context. Therefore, a lot of attention has been given to transferring concepts of game theory to the quantum realm hoping that this work would contribute to our understanding of this difficult field of research.

Quantum games are games in the standard sense but the approach allows for harnessing quantum phenomena during the course of the game [17, 18. The developed formalism can be also used in a more abstract sense regardless of its the quantum theoretical roots. Some classical game theoretical issues can be extended to allow for 
quantum strategies including cooperation and coordination problems. The set of quantum strategies is much larger than the set of classical ones and the presence of entanglement in the extended in such way games ("quantized") implies more complex behavior of agents than the one implied by "classical mixing" of strategies [16.

For the purpose of this paper, $N$-player quantum game can be defined as a quadruple

$$
\Gamma=(\mathcal{H}, \rho, \mathcal{S}, \mathcal{P}) .
$$

In this ordered list $\mathcal{H}$ is a Hilbert space, $\rho$ is a quantum state (i.e. a density matrix), $\mathcal{S}=\left\{S_{i}\right\}_{i=1}^{N}$ is the set of possible player's strategies and $\mathcal{P}=\left\{P_{i}\right\}_{i=1}^{N}$ is the set of payoff functions for the players. Quantum strategies $s_{i}^{\alpha} \in S_{i}$ are completely positive trace preserving (CPTP) maps. The payoff function of $i$-th player $P_{i}$ assigns to a given strategy profile i. e. a set of player's strategies $\left\{s_{j}^{\alpha_{j}}\right\}_{j=1}^{N}$ a real number - the payoff.

Usually, the set of strategies is limited to unitary operators and the payoff is determined via a measurement of the appropriate variables. A rich strategy sets often allows for some spectacular results. For example, it has been shown that if only one agent is aware of the quantum nature of the system, s/he will never lose in some types of quantum games [19. Moreover, it has been demonstrated that a player can cheat by appending additional qubits to the quantum system in question 20 . One can also study the impact of random strategies on the course of the game 21.

\section{QUANTUM GAMES ON 2D-LATTICE}

Let us consider a two-dimensional lattice with $M_{1} \times M_{2}$ nodes. Each node of the lattice is occupied by one player.

Our scheme consists of three steps. In the first step, each agent in the network is assigned an initial capital. In the second step, we create a quantum game setup for a selected agent and all his/hers nearest neighbors. This referrers to creation of an shared entangled state in standard Eisert quantization of games, or a creation of a shared coin state in a quantum Parrondo game. After the shared state is created, a quantum game is played by an agent with all of his/her neighbors.

In the case of periodic boundary conditions, we need only to concern ourselves with a five-player game, as every agent in a periodic lattice has four neighbors. In the non-periodic case, we must also study the three- and fourplayer game. Based on the results of the game and the game's payoff function, a possible capital change vector is obtained.

In the third step, the capital values of each player is updated using the capital change vectors from the previous step. This is done for every agent in the lattice. This process is repeated $N$ times to enhance the differences in capital behavior. This setup allows to study a number of different cases, as one can assign different strategy setups for every number of players.
For a single player (node) on a lattice we introduce the following scheme for playing entanglement assisted quantum game.

- The state $\left|\psi_{m}\right\rangle$ describing the game at node $m$ is used to describe a subspace of coins and a subspace of position for each players,

$$
\left|\psi_{m}\right\rangle \in \mathbb{C}^{K_{m}^{2}} \otimes \mathbb{C}^{K_{m}^{4}}
$$

where $K_{m}$ is the number of players playing with player at the node.

- The initial state of the game is given as

$$
\left|\psi_{m}(0)\right\rangle=|\phi\rangle \otimes\left(\bigotimes_{K_{m}}|00\rangle\right)
$$

where $|\phi\rangle$ is a multipartite state shared by $K_{m}$ players and the position registers for all players are prepared in the base state.

- Evolution operator is composed of the walker $W_{m}$ and the shift operator $S_{m}$, where

$$
\mathcal{W}=\bigotimes_{i=1}^{K_{m}} X\left(s_{i}\right) \otimes \mathbb{1}^{K_{m}^{4}}
$$

with each $X\left(s_{i}\right) \in S U(2)$ and

$$
\mathcal{S}=\sum_{i_{1}, \ldots, i_{K_{m}}}\left(\bigotimes_{k=1}^{K_{m}}\left|i_{k}\right\rangle\left\langle i_{k}\right|\right) \otimes\left(\bigotimes_{k=1}^{K_{m}} S^{-1^{k+1}}\right),
$$

where $S=\sum_{x}|x+1\rangle\langle x|$.

\section{UNIFORM ANALYSIS OF ENTANGLED QUANTUM GAMES}

\section{A. Prisoner's dilemma}

The quantum prisoner's dilemma game is defined as follows. Each player is sent a qubit and can locally operate on it, using any unitary operator $U \in S U(2)$. The initial state of the system is entangled:

$$
|\psi\rangle=J|0\rangle^{\otimes N}
$$

where $N$ is the number of players, $J$ is the entangling operator 18 .

$$
J=\frac{1}{\sqrt{2}}\left(\mathbb{1}^{\otimes N}+i \sigma_{x}^{\otimes N}\right) .
$$

After the players have applied their respective strategies, the untangling gate, $J^{\dagger}$, is applied to the system, hence the final state of the game is

$$
\left|\psi_{f}\right\rangle=J^{\dagger}\left(\bigotimes_{i=1}^{N} U_{i}\right) J|0\rangle^{\otimes N}
$$


where $U_{i}$ is the strategy of the $i$-th player. The payoff of the first player amounts to:

$$
\$_{A}=\sum_{i_{1} \ldots i_{N} \in\{0,1\} \times N} p_{i_{1} \ldots i_{N}}\left\langle\psi_{f} \mid i_{1} \ldots i_{N}\right\rangle,
$$

where $p_{i_{1} \ldots i_{N}}$ are numbers corresponding to the possible classical payoffs of the first player. We assign the payoffs in the way described in 22, Equation (23).

In the case of Prisoner's dilemma, the quantum version of the game utilizes an extended set of strategies, which includes unitary operators. In the case of our scheme one can consider three scenarios by restricting the set of strategies available for the players, namely

1. only classical strategies, i.e.

$$
\{C, D\}
$$

2. only quantum strategies from the set,

$$
\{H, Q, \Sigma\}
$$

3. classical strategies with one quantum strategy,

$$
\{C, D, H\},\{C, D, Q\},\{C, D, \Sigma\}
$$

where the unitary strategies are

- $C=\left(\begin{array}{ll}1 & 0 \\ 0 & 1\end{array}\right)$,

- $D=\left(\begin{array}{ll}0 & 1 \\ 1 & 0\end{array}\right)$,

- $H=\frac{1}{\sqrt{2}}\left(\begin{array}{cc}1 & 1 \\ 1 & -1\end{array}\right)$,

- $Q=\left(\begin{array}{cc}\mathrm{i} & 0 \\ 0 & -\mathrm{i}\end{array}\right)$,

- $\Sigma=\left(\begin{array}{cc}0 & 1 \\ -1 & 0\end{array}\right)$.

One should note that the strategy $H$ cannot be interpreted as a mixture of classical strategies as it operates on the normalized vectors, whilst the mixed strategy operates on the probabilities.

We perform 100 consecutive updates of the players' capital and we consider on orthogonal neighbors. During each update, the players on a central node play the game with five different players - one in which they are at the center of their four neighbors, and four where they are one of the neighbors for other central players. Players on edge nodes play four games at each step - one a fiveplayer game and the others four- or three-player games. A corner player participates in two four-player games and one three-player game. In each of the games a different set of entangled qubits is used.

The results obtained for the first case are shown in Figure 1 a. In it, we show the average capital of players on a $5 \times 5$ network averaged over all possible strategy combinations. As can be seen, the highest average capital gains are achieved by players closer to the edge of the lattice. The average capital of an agent in the lattice is equal to

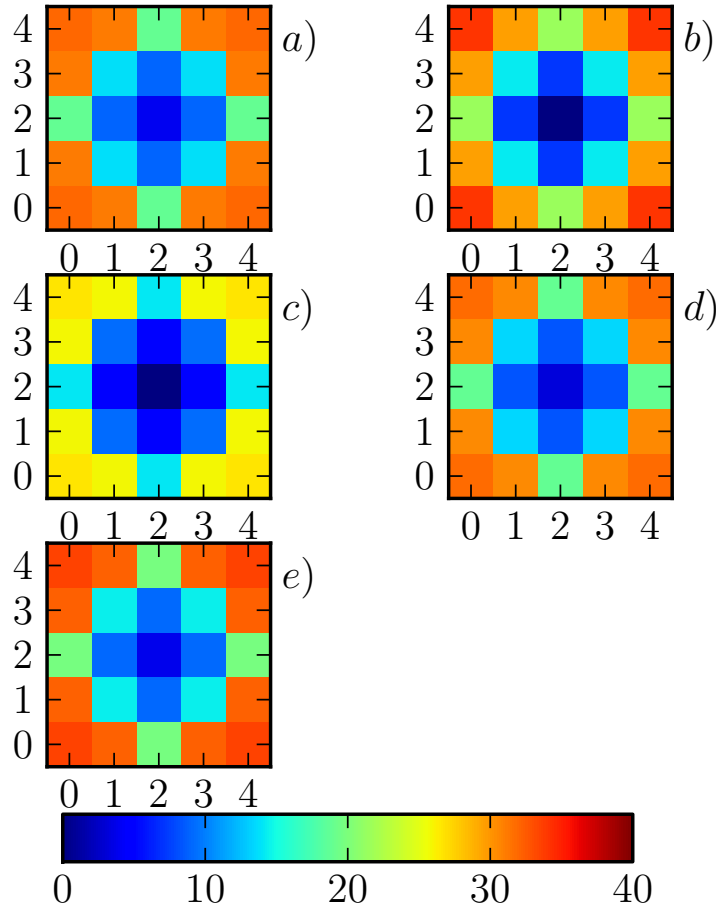

FIG. 1: Average capital distribution for the prisoner's dilemma game in the case of classical strategies (a), quantum strategies (b), classical strategies with addition of the quantum strategies $H, Q, \Sigma$ (c,d,e respectively).

19. The strategy set which yields the highest average capital of a player is $[(C, C, C),(C, C, C, C),(C, C, C, C, C)]$ and gives an average capital of 588.8. A strategy set $(A, B, C)$ means that the first player uses strategy $A$, the second strategy $B$ and the third strategy $C$. The capital distribution for this case is shown in Figure 2

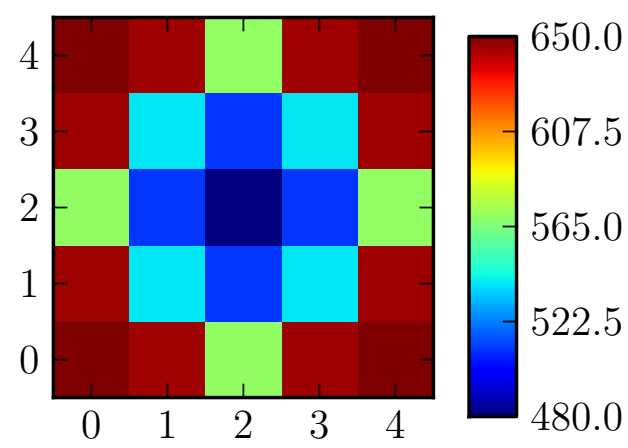

FIG. 2: Capital distribution for the prisoner's dilemma game in the case of classical strategies. The strategy set is $[(C, C, C),(C, C, C, C),(C, C, C, C, C)]$ and gives the highest average capital of agents of all strategy sets.

This figure also illustrates the cases where we added a quantum strategy to the classical ones.

The second case is illustrated in Figure $1 \mathrm{~b}$. In this case, similar to the classical one, highest capital gains are found for the edge players. Also, the overall capital dis- 
tribution is the same as in the purely classical case. The average capital of an agent in the lattice is equal to 17.8 . The strategy set which yields the highest average capital of a player is $[(\Sigma, \Sigma, \Sigma),(Q, Q, Q, Q),(\Sigma, \Sigma, \Sigma, \Sigma, \Sigma)]$ and gives an average capital of 588.8. The capital distribution for this case is shown in Figure 3

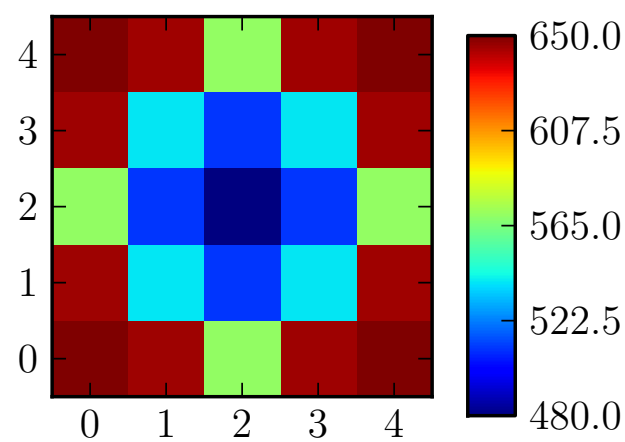

FIG. 3: Capital distribution for the prisoner's dilemma game in the case of quantum strategies. The strategy set is $[(\Sigma, \Sigma, \Sigma),(Q, Q, Q, Q),(\Sigma, \Sigma, \Sigma, \Sigma, \Sigma)]$ and gives the highest average capital of agents of all strategy sets.

We present the results for the third case in Figures 1 , $1 \mathrm{~d}$ and 1 for the possible strategies $\{C, D, H\},\{C, D, Q\}$ and $\{C, D, \Sigma\}$ respectively. The average capital gains are $-29.6,15.4$ and 28.2 respectively. As can be seen, the average capital gain with the added strategy $\Sigma$ is higher than in the purely classical case. The strategy set which gives the highest average capital gain of a player is $[(C, C, C),(C, C, C, C),(C, C, C, C, C)]$, the same as in the purely classical case. Hence, the capital distribution for this case is identical to that shown in Figure 2 and the average capital gain is equal to 588.8 .

\section{B. Cooperative Parrondo paradox}

Cooperative Parrondo's games were introduced by Toral 23 . The scheme is as follows. Consider an ensemble of $N$ players, each with his/hers own capital $C_{i}(t)$, $i=1,2, \ldots, N$. As in the original paradox, we consider two games, A and B. Player $i$ can play either game A or $\mathrm{B}$ according to some rules. The main difference from the original paradox is that probabilities of game B depend, in general, on the state of all players $j \neq u$. For simplicity, we only consider the case when the probabilities of winning at time $t$, depend only on the present state of the players. The game, by definition, is a winning one, when the average value of the capital

$$
\langle C(t)\rangle=\frac{1}{N} \sum_{i=1}^{N} C_{i}(t),
$$

increases with time.

There are several known approaches to quantization of Parrondo's games [24, 25. We model a cooperative quantum Parrondo's game as a multidimensional quantum walk $(\mathrm{QW})$ [26, 27. The average position of the walker along each axis determines each player's payoff. As in the classical case, we consider two games, $A$ and $B$. Similar to the classical case the probabilities of winning game $B$ depend on the state of the other players. A detailed introduction of the model of the game is presented in 28 .

The following two possible schemes of alternating between games $A$ and $B$ are considered

1. random alternation, denoted $A+B$

2. games played in succession $A A B B A A B B \ldots$, denoted $[2,2]$.

We focus our attention on the cooperative game in a regular lattice. We consider two types of boundary conditions for the lattice: periodic, allowing to simulate the behavior of an infinite lattice, and non-periodic to simulate the finite case.

With each agent on the network we associate a Hilbert space that consists of two components: the coin's Hilbert space and the position Hilbert space

$$
\mathcal{H}_{i}=\mathcal{H}_{c} \otimes \mathcal{H}_{\text {pos }}
$$

We introduce two base states in the single coin Hilbert space, the $|L\rangle$ and $|R\rangle$ states. These states represent the classical coin's heads and tails respectively.

When the agents play the game, we connect their respective Hilbert spaces using the tensor product

$$
\mathcal{H}_{G}=\bigotimes_{i=1}^{N} \mathcal{H}_{i}
$$

where $N$ denotes the total number of players participating in the game.

Game A is implemented using an operator performing a flip of a fair coin. The operator is given by

$$
U_{A}=\left(\begin{array}{cc}
\frac{1}{\sqrt{2}} & \frac{\mathrm{i}}{\sqrt{2}} \\
\frac{\mathrm{i}}{\sqrt{2}} & \frac{1}{\sqrt{2}}
\end{array}\right) .
$$

The game $\mathrm{B}$ is played as follows. An agent $i$ performs a flip of his coin using one of the unitary operators from the family

$$
U_{k}=\left(\begin{array}{cc}
\sqrt{\rho_{k}} & \sqrt{1-\rho_{k}} \mathrm{e}^{\mathrm{i} \theta_{k}} \\
\sqrt{1-\rho_{k}} \mathrm{e}^{\mathrm{i} \phi_{k}} & -\sqrt{\rho_{k}} \mathrm{e}^{\mathrm{i}\left(\theta_{k}+\phi_{k}\right)}
\end{array}\right) .
$$

Each operator in the set $\left\{U_{k}\right\}$ depends on the parameters: $\rho_{k}$ which is the classical probability that the coin does not change its state, and $\phi_{k}$ and $\theta_{k}$ are phase angles, which we assume to be $\phi_{k}=\theta_{k}=\pi / 2$ for all $k$. The choice of the probabilities, and thus an operator from the set $\left\{U_{k}\right\}$, depends on the number of neighbors and the number of winners and losers amongst the neighbors of an agent $i$.

We set the probabilities in game B to $\rho=0.5$ except for the case when all the other players have lost. In this case, we set it to $\rho=0.9$. 
After the coin flip, agent $i$ applies a move operator to his $\mathcal{H}_{i}$ space. The operator is given by

$$
U_{\text {pos }}=P_{r} \otimes S+P_{l} \otimes S^{\dagger},
$$

where $S$ is a shift operator in the position space, defined as $S|x\rangle=|x+1\rangle,|x\rangle$ denotes the current position of the walker. It follows from the definition of $S$ that $S^{\dagger}|x\rangle=$ $|x-1\rangle . P_{r}$ and $P_{l}$ denote projection operators on the $|R\rangle$ and $|L\rangle$ states of the coin respectively.

We study the behavior of the lattice for different sets $\left\{U_{k}\right\}$ of possible game B coin tosses. We chose those sets so that

1. a three and four player game shows paradoxical behavior,

2. game shows paradoxical behavior for three, four and five players

Furthermore, we study different initial states of players' coins: the separable state, the entangled GHZ state and the entangled $\mathrm{W}$ state.

The setup of the simulation is as follows. Each agent in the network plays a game with all of his/hers neighbors. The network is updated sequentially. The network is evolved for 1000 iterations. In the case of the $\mathrm{A}+\mathrm{B}$ game scheme, we average the results for 10 independent runs of the simulation.

Figure 4 shows the results of the simulation when the initial state of the coin is separable. Figures $4 \mathrm{a}$ and $4 \mathrm{~b}$ show the results of the simulation for the non-periodic network and Figures $4 \mathrm{c}$ and $4 \mathrm{~d}$ show the results for the periodic network. In the case of non-periodic network, the final structure of the network appears similar for both games: the only difference being the absolute value of capital of each agent. The same is true for the periodic network. Bar plots presented in Figure $4 \mathrm{e}$ shows the average capital gains of the players in a three, four and five-player game. The game $\mathrm{A}+\mathrm{B}$ shows paradoxical behavior only in the three-player case, whereas the $[2,2]$ game shows this kind of behavior in three and fourplayer games. As the five-player game is always a losing one and the network contains mostly agents playing the five player game, the average capital gain of an agent in the network is negative. In fact, almost every agent experiences a capital decrease. Figure $4 \mathrm{f}$ shows the average capital of an agent in all studied cases.

In the case of the GHZ state, the results are summarized in Figure 5. Figures 5a and 5b show the results of the simulation for the non-periodic network and Figures $5 \mathrm{c}$ and $5 \mathrm{~d}$ show the results for the periodic network. The capital distribution in the network differs significantly from the one obtained for the separable coin state. In this case, games $[2,2]$ and $\mathrm{A}+\mathrm{B}$ show paradoxical behavior in the case of three, four and five players as depicted in Figure 5e. Also, the value of average capital gain in each of these games is much higher than in the case of the separable coin state. This is reflected in the average capital gain of the entire network after 1000 iterations. As shown in Figure $5 \mathrm{f}$ the average capital gain of the network is much greater than in the separable case. This is due to the fact, that all games show paradoxical behavior. Therefore, the agents in the network always gain capital.

Finally, we show results for the $\mathrm{W}$ state in Figure 6 . Figures $6 \mathrm{a}$ and $6 \mathrm{~b}$ show the results of the simulation for the non-periodic network and Figures $6 \mathrm{c}$ and $6 \mathrm{~d}$. show the results for the periodic network. The capital distribution in the case of the $A+B$ resembles the GHZ case, whereas the distribution for the $[2,2]$ is flat. This is due to the fact that, the game does not show paradoxical behavior in the three player case, as game B is a winning one. Nevertheless, the paradox is observed for the four and five player $\mathrm{A}+\mathrm{B}$ games as shown in Figure $6 \mathrm{e}$. This leads to high average capitals of the network in the case of the $\mathrm{A}+\mathrm{B}$ game. As game [2,2] is a losing one for all studied number of players, the average capitals of the networks are always negative in this case as shown in Figure 6f

\section{CONCLUSIONS}

We introduced a general scheme for executing quantum games on regular lattices, which allows for the usage of entangled states and enables uniform analysis of different scenarios. To demonstrate the merits of the introduced scheme, we studied the quantum Parrondo effect and the quantum prisoner's dilemma game in regular lattices of agents.

The results for the prisoners dilemma game suggest that addition of the quantum strategy $\Sigma$ to the classical strategies: cooperation $(C)$ and defection $(D)$ gives a higher average capital gain of an agent than in the classical case.

In the case of Parrondo paradox, the games showing paradoxical behavior were modeled using quantum walks. We obtained results, showing that the average capital of an agent in the lattice grows in the following setups of the game:

- $[2,2]$ game on a periodic lattice with the GHZ coin state,

- $\mathrm{A}+\mathrm{B}$ game on a periodic lattice with the GHZ coin state,

- $[2,2]$ game on a non-periodic lattice with the GHZ coin state,

- $\mathrm{A}+\mathrm{B}$ game on a non-periodic lattice with the GHZ coin state,

- $\mathrm{A}+\mathrm{B}$ game on a periodic lattice with the $\mathrm{W}$ coin state,

- $\mathrm{A}+\mathrm{B}$ game on a non-periodic lattice with the $\mathrm{W}$ coin state.

The above results show that entanglement is a necessary condition for the lattice to gain capital as a whole. 


\section{ACKNOWLEDGMENTS}

Work by J. Sładkowski was supported by the Polish National Science Centre under the project number DEC2011/01/B/ST6/07197. Work by E. Pawela and J. Miszczak was supported by the National Science Centre under the project number DEC-2011/03/D/ST6/00413.

[1] M. E. J. Newman, Networks: An Introduction (Oxford University Press, 2010).

[2] R. Axelrod and W. Hamilton, Science 211, 1390 (1981).

[3] R. Axelrod, The evolution of cooperation: revised edition (Basic books, 2006).

[4] M. Nowak and R. May, Nature 359, 826-829 (1992).

[5] M. Tomassini, L. Luthi, and E. Pestelacci, International Journal of Modern Physics C 18, 1173 (2007), URL http://dx.doi.org/10.1142/S0129183107011212.

[6] N. Rosario, H. Mantegna, and E. Stanley, An Introduction to Econophysics: Correlations and Complexity in Finance (Cambridge University Press, 1999).

[7] P. Mirowski, More Heat than Light - Economics as Social Physics (Cambridge University Press, 1989).

[8] S. Galam, Sociophysics. A Physicist's Modeling of Psycho-political Phenomena (Springer, 2012).

[9] A. Khrennikov, Ubiquitous Quantum Structure: From Psychology to Finance (Springer, 2010).

[10] J. R. Busemeyer, Z. Wang, and J. T. Townsend, Journal of Mathematical Psychology 53, 220 (2009), URL http: //dx.doi.org/10.1016/j.jmp.2009.03.002.

[11] Q. Li, A. Iqbal, M. Chen, and D. Abbott, Physica A 391, 3316 (2012), URL http://dx.doi.org/10.1016/j. physa.2012.01.048.

[12] E. Pawela and J. Sładkowski, Physica A 392, 910 (2013), URL http://dx.doi.org/10.1016/j.physa. 2012.10.034

[13] J. Sładkowski, Physica A 324, 234 (2002).

[14] E. M. Pothos, G. Perry, P. J. Corr, M. R. Metthew, and J. R. Busemeyer, Personality and Individual Differences 51, 210 (2011).

[15] G. P. Harmer and D. Abbott, Statistical Science 14, 206 (1999).

[16] M. Osborne and A. Rubinstein, A course in game theory (The MIT press, 1994).

[17] E. W. Piotrowski and J. Sladkowski, arXiv:quant- ph/0211191 (2002), Int. J. Theor. Phys. 42 (2003) 1089, URL http://arxiv.org/abs/quant-ph/0211191.

[18] S. Benjamin and P. Hayden, Physical Review A 64, 030301 (2001), URL http://link.aps .org/doi/10. 1103/PhysRevA.64.030301.

[19] J. Eisert, M. Wilkens, and M. Lewenstein, Physical Review Letters 83, 3077 (1999), URL http://link.aps. org/doi/10.1103/PhysRevLett.83.3077

[20] J. Miszczak, P. Gawron, and Z. Puchała, Quantum Information Processing pp. 1571-1583 (2012), URL http: //dx.doi.org/10.1007/s11128-011-0322-2

[21] J. Košík, J. A. Miszczak, and V. Bužek, Journal of Modern Optics 54, 2275 (2007), ISSN 0950-0340, URL http://www.tandfonline.com/doi/abs/10.1080/ 09500340701408722 .

[22] A. P. Flitney and L. C. Hollenberg, Physics Letters A 363, 381 (2007).

[23] R. Toral, arXiv:cond-mat/0101435 (2001), fluctuations and Noise Letters, 1, L7 (2001), URL http://arxiv. org/abs/cond-mat/0101435

[24] A. Flitney, J. Ng, and D. Abbott, Physica A: Statistical Mechanics and its Applications 314, 35 (2002), ISSN 0378-4371, URL http://wWw.sciencedirect.com/ science/article/pii/S0378437102010841.

[25] P. Gawron and J. A. Miszczak, Fluctuation and Noise Letters 5, L471-L478 (2005), URL http://dx.doi.org/ 10.1142/S0219477505002902.

[26] A. P. Flitney, D. Abbott, and N. F. Johnson, Journal of Physics A: Mathematical and General 37, 7581 (2004), ISSN 0305-4470, 1361-6447, URL http://iopscience. iop.org/0305-4470/37/30/013

[27] C. Chandrashekar and S. Banerjee, Physics Letters A 375, 1553 (2011).

[28] Ł. Pawela and J. Sładkowski, Physica D 256-257, 51 (2013), URL http://dx.doi.org/10.1016/j.physd. 2013.04.010. 


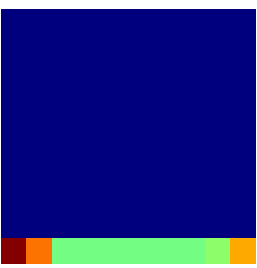

(a) $[2,2]$ non-periodic lattice.

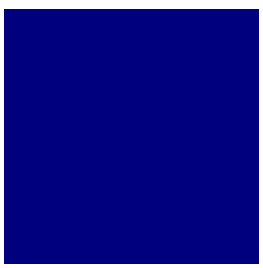

(c) $[2,2]$ periodic lattice.

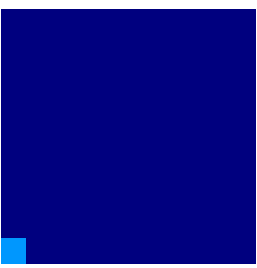

(b) $\mathrm{A}+\mathrm{B}$

non-periodic

lattice.

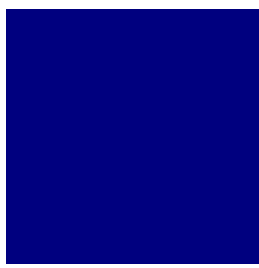

(d) A+B periodic lattice.
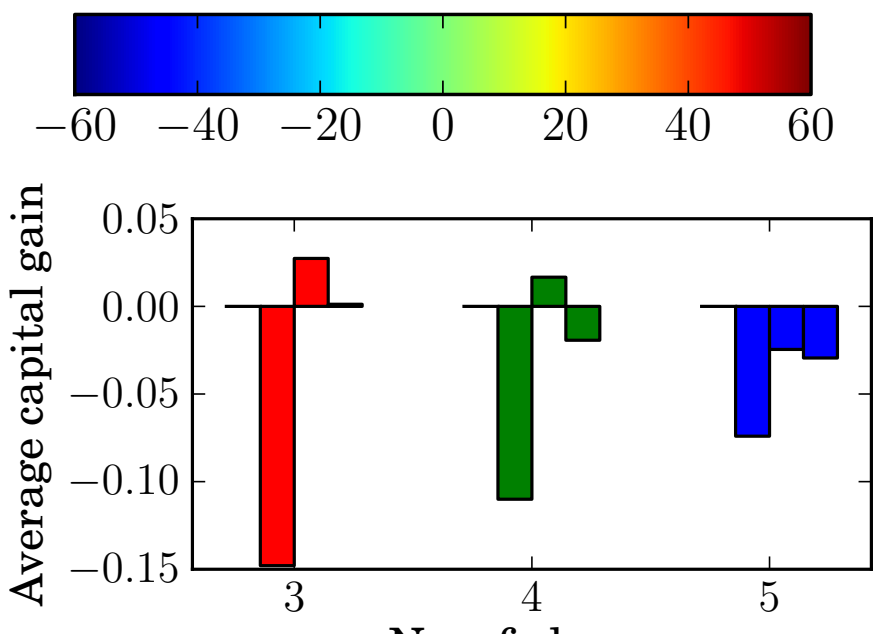

No. of players

(e) Average payoff as a function of the number of players. The bars show the payoff for game $\mathrm{A}$, game $\mathrm{B}$, game $[2,2]$ and game $\mathrm{A}+\mathrm{B}$ going from left to right.

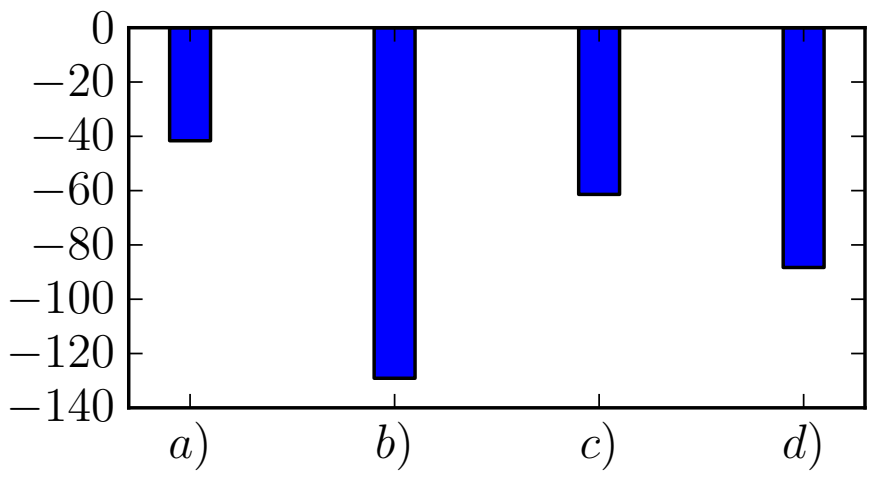

(f) The average capital of the networks shown in Figures $4 \mathrm{a} 4 \mathrm{~b}$ $4 \mathrm{c} 4 \mathrm{~d}$

FIG. 4: The state of the network for the $[2,2]$ game on non-periodic lattice (a)), A+B game on non-periodic lattice (b)), $[2,2]$ game on periodic lattice (c)) and $\mathrm{A}+\mathrm{B}$ game on periodic lattice (d)) after 1000 iterations. The initial coin state is the separable state. Figure e) shows the payoff for games $\mathrm{A}, \mathrm{B},[2,2]$ and $\mathrm{A}+\mathrm{B}$ as a function of the number of players. Figure $f$ ) shows the average capital gain of an agent in the network after 1000 iterations. 


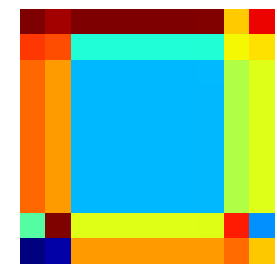

(a) $[2,2]$ non-periodic lattice.

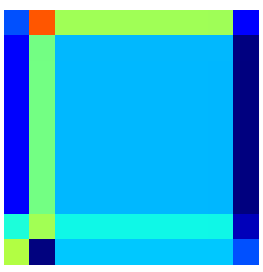

(c) $[2,2]$ periodic lattice.

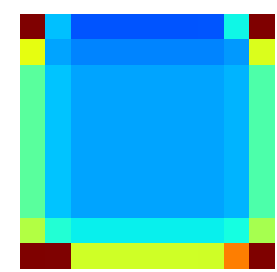

(b) $\mathrm{A}+\mathrm{B}$ non-periodic lattice.

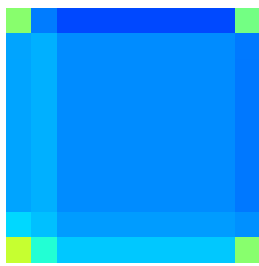

(d) A+B periodic lattice.
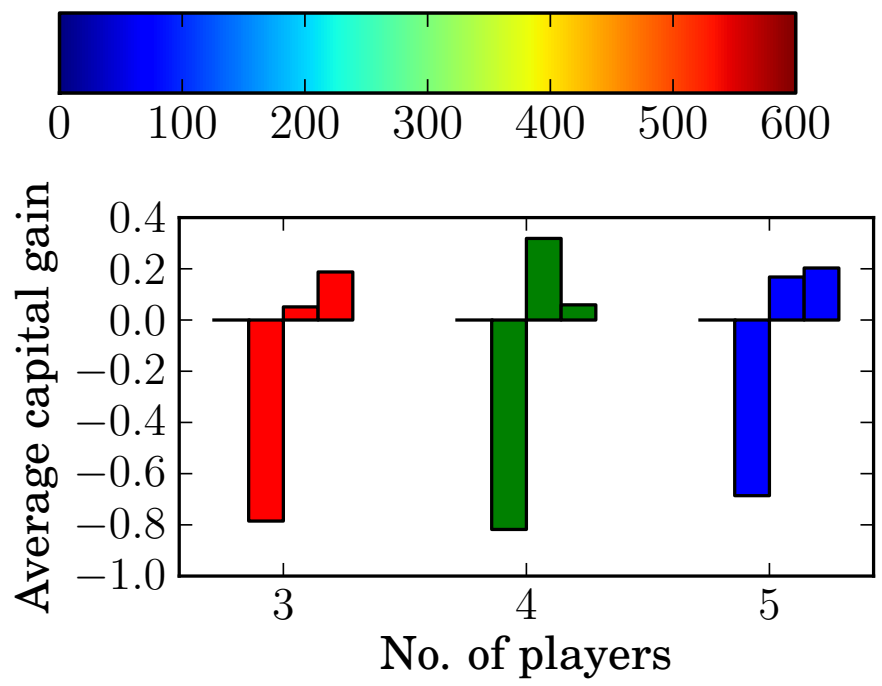

(e) Average payoff as a function of the number of players. The bars show the payoff for game $\mathrm{A}$, game $\mathrm{B}$, game $[2,2]$ and game $\mathrm{A}+\mathrm{B}$ going from left to right.

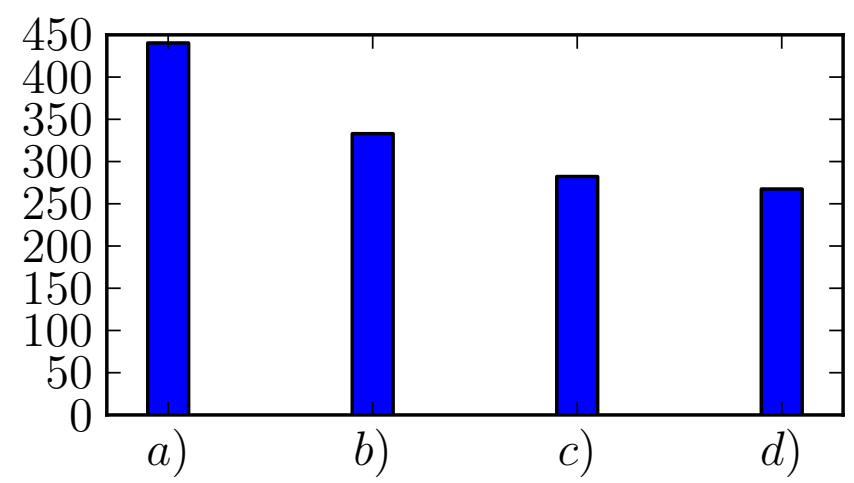

(f) The average capital of the networks shown in Figures $5 \mathrm{a}, 5 \mathrm{~b}$ $5 \mathrm{c} 5 \mathrm{~d}$

FIG. 5: The state of the network for the $[2,2]$ game on non-periodic lattice (a)), A+B game on non-periodic lattice (b)), $[2,2]$ game on periodic lattice (c)) and $A+B$ game on periodic lattice $(\mathrm{d}))$ after 1000 iterations. The initial coin state is the GHZ state. Figure e) shows the payoff for games $\mathrm{A}, \mathrm{B},[2,2]$ and $\mathrm{A}+\mathrm{B}$ as a function of the number of players. Figure f) shows the average capital gain of an agent in the network after 1000 iterations. 


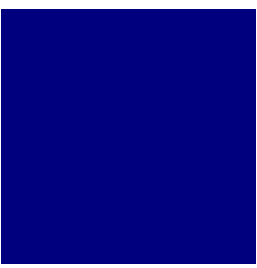

(a) $[2,2]$ non-periodic lattice.

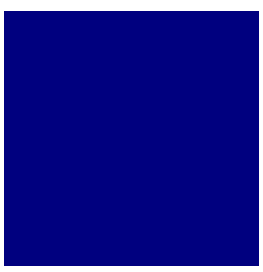

(c) $[2,2]$ periodic lattice.

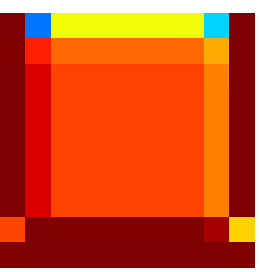

(b) $\mathrm{A}+\mathrm{B}$

non-periodic

lattice.

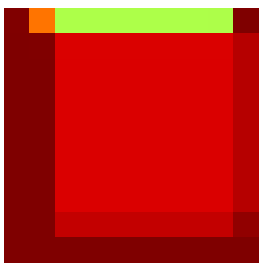

(d) A+B periodic lattice.
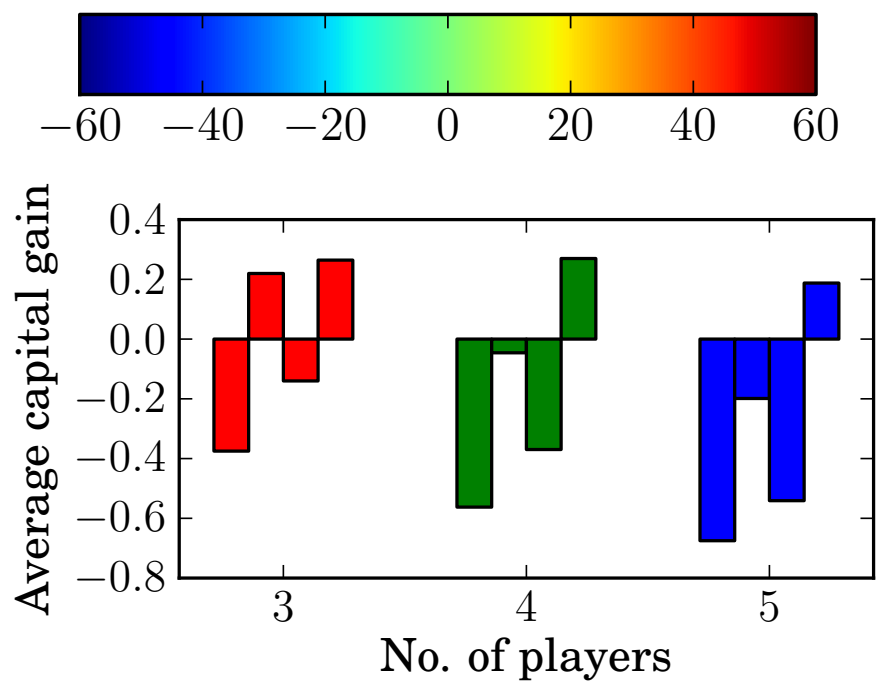

(e) Average payoff as a function of the number of players. The bars show the payoff for game $\mathrm{A}$, game $\mathrm{B}$, game $[2,2]$ and game $\mathrm{A}+\mathrm{B}$ going from left to right.

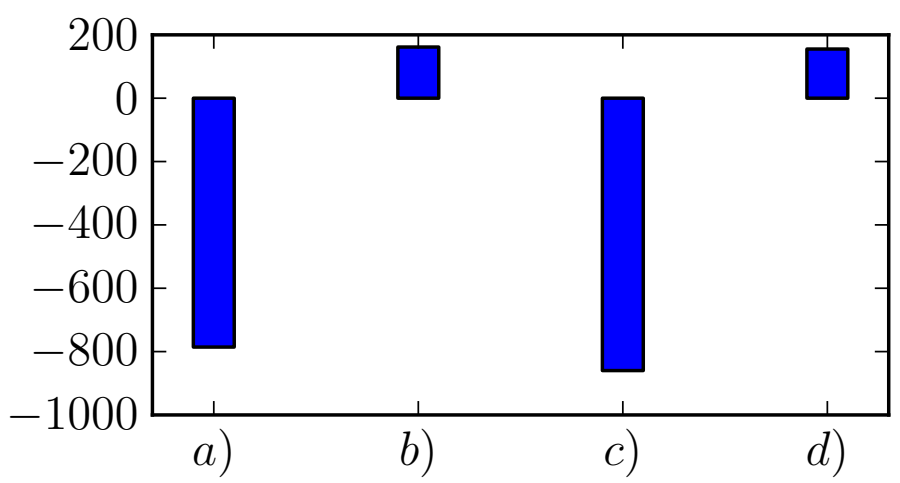

(f) The average capital of the networks shown in Figures $6 \mathrm{a} 6 \mathrm{~b} 6 \mathrm{c}$. $6 \mathrm{~d}$

FIG. 6: The state of the network for the $[2,2]$ game on non-periodic lattice (a)), A+B game on non-periodic lattice (b)), $[2,2]$ game on periodic lattice (c)) and $A+B$ game on periodic lattice $(\mathrm{d}))$ after 1000 iterations. The initial coin state is the $\mathrm{W}$ state. Figure e) shows the payoff for games $\mathrm{A}, \mathrm{B},[2,2]$ and $\mathrm{A}+\mathrm{B}$ as a function of the number of players. Figure $\mathrm{f}$ ) shows the average capital gain of an agent in the network after 1000 iterations. 